


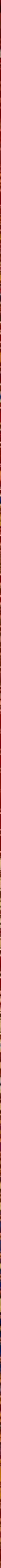








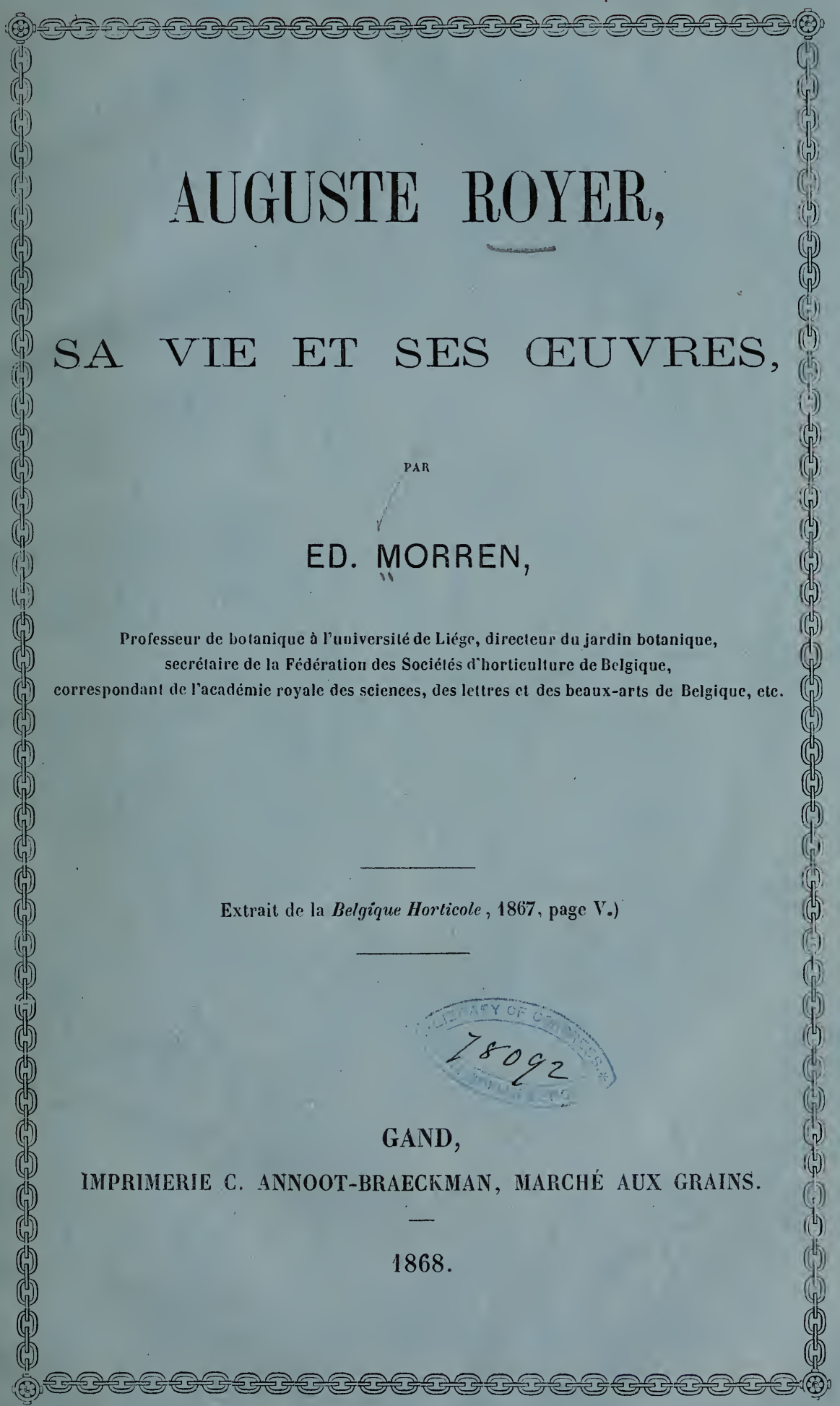




$$
\frac{5 B 6^{3}}{R 7 M^{6}}
$$




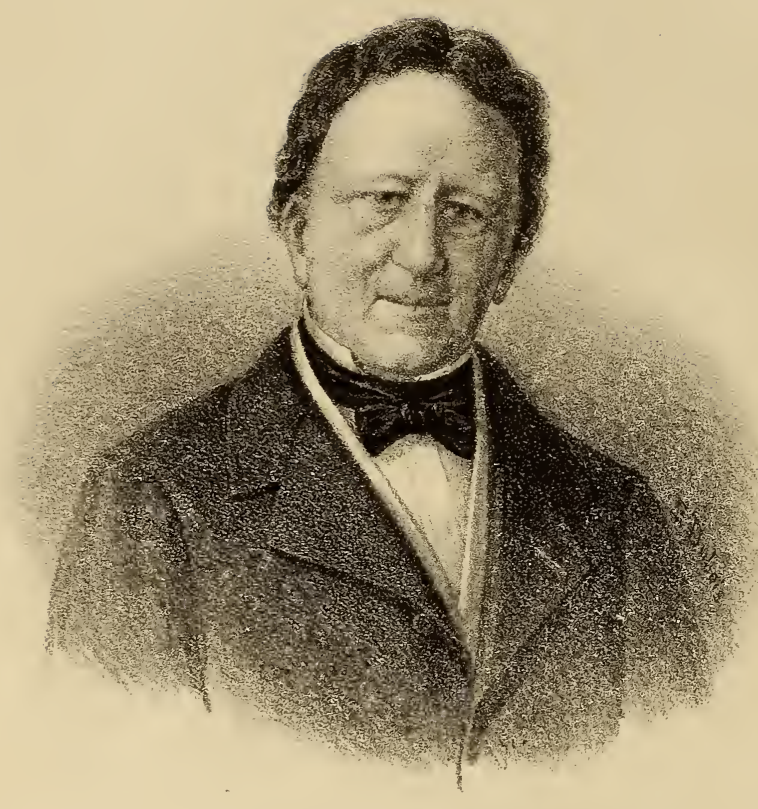

Aug. POYER. 


\title{
PROLOGUE
}

\author{
A LA MÉMOIRE DE
}

\section{AUGUSTE-PHILIPPE-ANTOINE ROYER.}

\author{
$1796-1867$.
}

C'est comme un devoir de piété filiale que nous remplissons en consacrant ce dix-septième volume de la Belgique horticole à la mémoire d'Auguste Royer. Sa mort nous a produit une douloureuse émotion qui se réveille au moment où nous prenons la plume pour tracer ces lignes.

Royer était aimé de ceux qui ont vécu autour de lui. Tous ceux qui ont été admis dans la communion de ses pensées et dans l'intimité de sa vie, ont pu apprécier l'élévation de son intelligence qui n'était égalée que par la bonté de son cour.

Nous avons eu le bonheur, bien jeune encore, et au début de notre carrière de connaitre Auguste Royer : c'était le 3 mai $185 \% 9$, à l'assemblée générale de tous les délégués de l'horticulture belge venus à Malines, pour fonder la Fédération des Sociétés d'horticulture. Cette belle et féconde institution fut établie sous son inspiration. Il présida à ses débuts et il ne cessa jusqu'à la fin de ses jours de lui consacrer sa judicieuse expérience. Il aimait cette Fédération, témoignage de la puissance de notre horticulture Belge et de la sympathie qui relie entre eux tous ses représentants, et il usa de la légitime influence qu'il exerçait pour lui 
faciliter tout ce qui était nécessaire à son installation. L'œuvre était nouvelle : elle répondait sans doute à des aspirations générales, mais il était néanmoins difficile de la réaliser : elle aurait pu acquérir un certain caractère de centralisation incompatible avec les traditions d'indépendance qui ont pénétré tous les esprits en Belgique. Royer sut éviter cet écueil, et, à son appel, toutes les Sociétés locales se groupèrent en un seul faisceau sans rien sacrifier de leur autonomie(1).

Nous avons eu l'honneur de seconder Auguste Royer en qualité de Secrétaire et bientòt les relations les plus intimes se sont établies entre nous. Ainsi, nous nous sommes trouvé en position de reconnaitre l'élévation de son intelligence, la profondeur de ses jugements, la rectitude de ses appréciations, son infatigable activité et l'abnégation avec laquelle il se consacrait au bien public, son expérience des hommes et des choses et ses vastes connaissances: ainsi encore il se fait que nous croyons pouvoir apporter ici notre propre témoignage parmi toutes les manifestations qui se sont produites quand est venu le terme de sa carrière si bien remplie.

Les manifestations de la haute estime publique n'ont pas manqué à la mémoire d'Auguste Royer. "Il était un de ces hommes rares, disait un de ceux qui ont pu le mieux l'apprécier, grand par le cœur, dévoué à la chose publique, toujours prèt à obliger et à donner son concours aux bonnes choses : la Belgique lui doit en grande partie la renaissance et la prospérité de plusieurs de ses industries et c'est à lui surtout qu'elle est redevable du développement merveilleux de son horticulture économique : ce qu'il a dépensé de zèle, de dévouement désintéressé et d'intelligence pour donner une impulsion féconde à cette grande branche du travail national, il n'y a que ceux qui ont eu la bonheur d'avoir des relations suivies avec lui, qui peuvent l'apprécicr. Sa modestic égalait son activité et c'est à peine si ceux mêmes qui jouissent des

(1) Voyez la circulaire du 11 juin 1859 insérée dans le premier volume du Bulletin de la Fédération. 
résultats de son initiative se doutent du labeur incessant qu'il s'est imposé pour les produire. "

Ces lignes sont de M. Bellefroid, directeur-général de l'agriculture et du commerce au ministère de l'intérieur.

L'horticulture belge a déjà fait entendre la voix de la reconnaissance sur la tombe d'Auguste Royer. Un de ses collègues et de ses meilleurs amis, M. Ferdinand Kegeljan, Secrétaire de la Société royale d'horticulture de Namur et membre du bureau de la Fédération, s'est exprimé en ces termes au bord de la tombe de notre digne et regretté président.

\section{DISCOURS DE M. KEGELJAN.}

J'obéis aux sentiments de l'amitié autant qu'à un douleureux devoir, en venant, au nom de la Société d'horticulture de Namur, adresser un dernier adieu à celui qui fut son président et l'un de ses membres les plus actifs.

Quoique absorbé déjà par le soin de ses affaires et par les devoirs que lui imposaient ses diverses fonctions, M. Royer savait trouver encore des heures de loisir, qu'il consacrait aux arts et aux sciences.

L'horticulture était surtout l'objet de ses prédilections.

Il fut fondateur et président de la Société Van Mons, et président de la Commission royale de Pomologie. - Il déploya en ces diverses qualités ce zèle et cette intelligence qui caractérisaient tous ses actes.

Quand se forma la Fédération des Sociétés d'horticulture de Belgique, il fut encore appelé à l'honneur de la présider et ses collègues le confirmèrent chaque année unanimement dans ces fonctions, rendant ainsi un éclatant hommage à son caractère et à son aptitude.

Ses mérites ne furent pas moins appréciés à l'étranger, et un grand nombre de Sociétés tinrent à honneur d'inscrire son nom parmi ceux de leurs membres correspondants.

L'agriculture fut également l'objet de ses soins et de ses études. Là encore son dévouement et son bon jugement le désignaient à ses col. lègues, qui l'appelèrent à la direction du Comice agricole de Namur, et plus tard, à la présidence de la section de Namur dans la Société agricole de la province, dont il était déjà vice-président.

Lorsque, en 1865 , il résigna ces dernières fonctions, l'assemblée générale, voulant lui témoigner sa reconnaissance, lui conféra le titre de président d'honneur.

Il serait trop long d'énumérer ici les services que M. Royer rendit à l'horticulture et à l'agriculture. 
Doué d'une imagination féconde, d'un grand esprit d'initiative et d'une activité infatigable, il sut imprimer une vive impulsion aux travaux des nombreuses associations dont il était chef ou membre.

Il s'était créé partout des relations en vue de rechercher les nouveautés dont pouvaient s'enrichir nos cultures et prenait un grand plaisir à les distribuer. Sous ce rapport encore il a rendu de notables services.

La bonté de son cœur, la loyauté de son caractère étaient connues de tous et un même sentiment de reconnaissance, d'affliction et de regrets nous réunit aujourd'hui autour de ses restes mortels.

Comme époux, comme père, comme ami, M. Royer peut être cité pour modèle, et ceux qui ont vécu dans son intimité ont pu voir combien il trouvait de bonheur et savait en répandre autour de son foyer.

Eprouvé depuis plus de quatre ans par une cruelle maladie, la patience et la résignation ne lui ont pas un seul instant fait défaut; il s'efforçait, au contraire, de cacher ses souffrances, afin de ne pas attrister ceux qui l'entouraient; il les oubliait souvent, pour s'enquérir de ses amis et de ses connaissances, avec cette sollicitude et cette bienveillance qui jamais ne l'abandonnaient.

Une vie si bien remplie a sans doute déjà trouvé sa récompense dans un monde meilleur. Puisse cette douce confiance, ainsi que le témoignage des regrets de nombreux amis, apporter quelque soulagement à la douleur d'une famille dont il était le chef bien-aimé.

Adieu, cher et vénéré collègue et ami, nous ne te verrons plus, hélas, parmi nous, mais nos cœurs seront toujours avec toi.

Royer s'est particulièrement adonné à la Pomolngie, cette branche si importante de l'horticulture économique. Il recherchait les résultats pratiques et directs de nature à augmenter la fortune publique et le bien-être des populations. Il a écrit les résultats de ses études dans les huit grands volumes des Annales de la pomologie belge et étrangère et dans le Bulletin de la Sociélé Van Mons.

Augriste Royer ne doit pas être seulement considéré comme pomologiste et horticulteur : il remplit des fonctions publiques et sut donner un élan remarquable à des industries nouvelles dont il a doté son pays. Ce côté important de son existence est particulièrement mis en relief dans le discours qui fut prononcé à ses funérailles par M. Dury, président du Conseil provincial de Namur. 


\section{DISCOURS DE M. DURY, PRÉSIDENT DU CONSEIL PROVINCIAL.}

De nombreuses sympathies et d'ineffaçables regrets viennent se mêler aux douleurs de l'honorable famille qui pleure celui quc nous avons aimé avec elle, notre digne ami, Auguste Royer, enlevé à sa tendresse et à notre attachement.

Témoin de ses vertus et de ses ouvres; durant plus de vingt ans, honoré de sa confiance et confident des sentiments et des idées qui ont été la règle de tous les actes de sa vie, j'accomplis un devoir, un devoir bien pénible, mais que je considère comme sacré, en venant, dans ce triste moment, rendre un dernier hommage à sa mémoire vénérée.

Lorsqu'un homme simple, droit, bon et généreux, pur de toute défaillance, disparaît de ce monde, c'est un devoir de signaler à la société la perte qu'elle a faite.

Entré de bonne heure dans la carrière commerciale et industrielle, Monsieur Royer a été un type d'ordre, d'exactitude et de délicate loyauté.

Sa remarquable intelligence et son indomptable activité devaient le conduire à des entreprises d'une importance peu commune; et en effet, il fut au premier rang parmi les fondateurs des plus grands établissements industriels de cette province.

Je parle surtout de ce vaste établissement de Floreffe qui a lutté pendant longtemps et qui ne cesse de rivaliser sur tous les marchés du monde avec les plus puissantes fabriques de glaces du continent.

Nous le trouvons au nombre des premiers organisateurs de cette colossale entreprise : - c'est en son nom qu'a été acquis le terrain sur lequel elle s'élève. C'est sous sa présidence et avec le concours de son énergie qu'elle s'est développée et qu'elle a atteint le degré de prospérité où nous la voyons s'élever.

Nous le rencontrons encore à l'origine de la création des verreries d'Herbatte qui prennent aujourd'hui une nouvelle importance par la formation de la puissante association réunissant en ses mains toutes les verrerics Namuroises.

Dans ces grandes créations, une large part fut faite à la bienfaisance. Il ne se contenta pas d'offrir du travail à une nombreuse population ouvrière, le bien-être de ses travailleurs devint l'objet de son intelligente sollicitude. Il les aidait à se ménager des ressources, à se créer une position, il veillait assidûment à leurs besoins moraux et religieux.

Quelle que fût la multiplicité des détails d'affaires dans lesquels s'est trouvé engagé M. Royer, jamais il n'en a été absorbé. Sa prodigieuse activité lui a toujours fait trouver au milieu des occupations les plus compliquées, un temps précicux, qu'il consacrait avec un rare bonheur 
à la culture d'une intelligence d'élite. Il trouvait du temps pour la lecture. Il lisait prodigieusenent et son heureuse ménoire accumulait d'inépuisables trésors de connaissances. Peu d'hommes sont parvenus à en acquérir d'aussi étendues. J'en appelle à ceux qui l'ont connu intimement, car il n'en faisait pas étalage.

Il était penseur, et les connaissances variées qu'il avait réunies, devenues l'aliment de ses méditations, en avaient fait un de ces hommes excellemment judicieux chez qui tout est pris à l'exacte mesure et rien n'est exagéré.

Est-il besoin de dire qu'il fut modéré et indulgent?

Oui, modéré et indulgent; mais jamais faible et capable de céder les droits de la vérité et de la justice.

Il s'était livré à une étude profonde de nos institutions. Il lcs connaissait bien et y était inviolablement attaché : il les considérait comme la meilleure garantie de toutes les existences et de toutes les opinions honnêtes et modérées. Aussi, dans la part qu'il a prise à nos luttes politiques, il ne s'est jamais proposé que de les défendre, et, avec elles, de sauvegarder les précieuses libertés qu'elles protégent.

M. Royer a dignement servi son pays dans les fonctions publiques dont il a été investi.

Il était un des membres les plus anciens, les plus assidus et les plus laborieux de la chambre de commerce, et il avait antérieurement fait aussi partie du tribunal de commerce.

Elu menbre du conseil provincial en 1848, il n'a pas cessé depuis cette époque de faire partie de cette assemblée et d'y apporter, avec l'appréciation parfaite des intérêts de ses commettants, l'esprit de suite et de persévérance qu'il savait allier à une constante modération et faire servir au triomphe de ce qu'il regardait comme juste et utile.

Beaucoup d'importantes résolutions ont été dues à une initiative à laquelle nous le trouvons associé. Je me borne à rappeler celles qui ont été le point de départ des sollicitations et des démarches actives de l'administration provinciale qui ont abouti à l'institution de l'entrepôt de douanc dont la station de Namur est aujourd'hui pourvue, et à l'accélération des travaux actuellement très-avancés que réclamait la navigation de la Meuse.

Il a présidé longtemps la commission du Conseil à laquelle il était attaché, et longtemps aussi il a rempli les fonctions délicates et laborieuses de la présidence du conseil de milice.

Ricn de ce qui pouvait contribuer au bien public ne le trouva indiffé-. rent.

La Société forestière et agricole, qui a rendu de véritables services à notre province, le comptait parmi ses membres. Il en était le viceprésident.

En donnant aux progrès de l'agriculture toute l'importance qu'ils mé- 
ritent, il s'était attaché avec plus d'intérêt et d'ardeur à stimuler le perfectionnement de la culture des jardins.

Je ne saurais peut-être, disait-il quelquefois, bien préciser ce que produit un hectare de terrain employé à la formation d'un verger et d'un jardin, mais je sais qu'il suffit à faire vivre dans l'aisance une famille qui en sait tirer parti. C'est le travail le mieux rétribué. - Partant de cette idée, c'était surtout à encourager l'horticulture et l'arboriculture, qu'il avait consacré les loisirs de ses dernières années.

Il est devenu le fondateur et le président de la Société d'horticulture, - le fondateur et le président de la commission royale de Pomologie, qui compte maintenant des correspondants dans toutes les parties du monde, - le promoteur et l'organisateur du congrès de pomologie, l'un des fondateurs des écoles d'horticulture et le président du jury d'examen de ces écoles.

Appréciant son dévouement et la valeur de ses services désintéressés, le Roi le nomma en 1854 chevalier de son Ordre.

Une vie si bien employée méritait cette noble récompense; mais il en méritait d'autres et il lui en fut accordé de plus douces et plus précieuses. Il s'est vu revivre dans un fils digne de lui; il a vu ses enfants heureux et ses derniers ans entourés de leur tendresse et de leurs soins affectueux. Il a béni deux générations de petits-enfants.

Enfin dans les derniers jours de souffrances d'une maladic hélas! bien longue et bien cruelle, il a trouvé Dieu ! Dieu, dont jamais il n'avait été séparé.

Sa piété, sans faste comme sans respect humain, ne s'était jamais démentie et sa foi religieuse, précieusement conservée par la pratique des devoirs qu'elle impose, a été son soutien, sa force et sa consolation. Arrivant au terme de ses souffrances avant d'avoir cessé de vivre, il s'est éteint doucement. Déjà il reposait dans le sein du divin protecteur en qui il avait mis son espoir.

Cet espoir est le nôtre.

Ami cher et regretté, Dieu vous a recueilli, parce que vous avez fait le bien, parce que vous avez cru et espéré en lui.

Puisse cette consolante confiance, puisse l'hommage mérité que nous rendons ici à votre mémoire, adoucir du moins les larmes d'une famille inconsolable qui ne cessera de vous pleurer jusqu'à ce que vous lui soyez rendu dans l'immortelle patrie, dans la vie nouvelle ou vous continuez à l'aimer.

Adieu Royer ! - Adieu.

Auguste -Philippe-Antoine Royer esı né à Namur le 26 janvier 1796 et mort dans la même ville le $1^{\mathrm{r}}$ octobre 1867 .

En 1846 et 1847 il a participé, en qualité de membre du 
comité de Namur, au mouvement économique qui s'était manifesté sous l'impulsion de Charles de Brouckere en faveur de la liberté commerciale. Membre du Congrès des économistes en 1847, il a pris une part notable aux travaux des sections. Il a collaboré fort activement au Congrès agricole de 1848 .

En 1849, il a publié une brochure assez volumineuse sur les expositions agricoles et leur utilité(1) et en 1850 un rapport trèsintéressant sur la durée des baux à fermes et l'amélioration de la propriété agricole(2).

On lui doit aussi un petit livre intéressant sur les vignobles et les vins $(3)$.

Il a collaboré fort activement dans plusieurs publications agricoles, pomologiques et horticoles, notamment les Annales de pomologie belge et étrangère, les Bullelins de la Fédération des Sociétés d'horticulture de Belgique, l'Agronome, journal de la Société agricole et forestière de la province de Namur, le Bulletin du Congrès international de pomologie qui a eu lieu à Namur au mois de septembre 1862, les Bulletins de la Société Van Mons, etc.

Il était en relations constantes avec le département de l'intérieur qui le consultait sur beaucoup de questions horticoles : plusieurs mémoires manuscrits écrits par lui existent dans les archives de ce département.

Il entretenait une correspondance suivie avec les horticulteurs français et américains. C'est par le moyen de ses correspondances en Amérique qu’il a pu importer et naturaliser en Belgique un grand nombre de variétés de Vignes.

Il fut nommé chevalier de l'Ordre de Léopold le 28 mars $183 ̈ 4$.

On le compte au nombre des membres fondateurs de la Société agricole et forestière de la province de Namur, dont il fut élu pre-

(1) Recherches sur l'utilité des expositions agricoles, brochure in-80 de 44 pages. Namur 1849.

(2) Rapport de la Commission du Comice sur les baux à fermes. Bruxelles, chez G. Stapleaux, 1850.

(3) Les vins; Etudes sur ceux qui se consomment en Belgique, in-12 de 100 pages. Bruxelles, $18: 32$. 
mier vice-président à son origine, le 28 mai 18ว9. Il contribua, par sa légitime expérience, à décider cette Société à créer un journal, organe de ses travaux agricoles. Tous les intérêts de la ville et de la province de Namur trouvèrent en lui un défenseur éclairé et dévoué. Il a publié un mémoire remarquable en faveur de l'industrie des soudes et il a contribué pour une certaine part à la suppression des remparts qui étreignaient la ville.

A l'ouverture de l'assemblée générale de la Fédération des Sociétés d'horticulture de Belgique qui a eu lieu à Bruxelles le 22 décembre 1867, M. F. de Cannart-d'Hamale, sénateur, vice-président de l'association, a prononcé l'éloge de celui que l'assemblée était hahituée à voir à sa tète. Voici cet excellent discours :

\section{Messieurs,}

Voici pour la quatrième fois que j'aborde ce fauteuil, mais malheureusement, je n'ai plus aujourd'hui, pour justifier cet honneur, l'excuse d'une indisposition grave de notre cher et digne président.

Hélas, le ciel nous l'a ravi, et c'est pour rendre hommage à sa mémoire, c'est pour déposer sur sa tombe à pcine fermée, un dernier gage d'affection et de regret, que je viens occuper, une fois encore, des fonctions qu'il savait exercer avec tant de talent et tant de bienveillance.

Permettez-moi, Messieurs, pour honorer sa mémoire, de remonter à une époque où un homme, alors ministre de l'intérieur et comprenant les grands intérêts de notre chère et belle patrie, conçut l'idée, et fit l'essai d'une exposition nationale d'agriculture.

C'était en 1847 que ce premier essai fut fait, et ce fut en 1848 que cette grande et noble institution qui exerça une si heureuse influence sur notre industrie agricole et horticole, est entrée jusque dans les entrailles du pays.

Ce fut dans ces luttes paisibles et fructueuses que je rencontrai pour la première fois M. Auguste Royer, alors qu'il fit ses premières armes et qu'il sut s'acquitter de la mission qui lui était confiée, avec tout le zèle, tout le dévouement et toute l'intelligence dont il n'a cessé depuis de nous donner des preuves nombreuses.

Successivement membre du jury des expositions nationales des produits de l'agriculture et de l'horticulture qui eurent lieu en 1847 et 1848 et membre du comité chargé de la partie pomologique de ces expositions, ce fut à lui que ses collègues confièrent la rédaction du rapport à adresser au gouvernement. 
C'esł dans ce rapport, Messieurs, daté du 16 septembre 1848, que nous rencontrons les premières données des mesures si utiles qui ont été prises, plus tard, en faveur de la culture des arbres fruitiers; ear c'était bien là la spécialité de feu notre digne président, et c'est dans ce même rapport qu'il appela toute l'attention du gouvernement sur l'utilité d'eneourager la eulture en grand des fruits de toutes espèces, afin d'alimenter, disait il, les marchés de nos villes, de procurer au peuple un supplément de substances alimentaires et de pousser en même temps à l'exportation toujours croissante de nos fruits à l'étranger.

Notre très-regretté président insista également auprès du gouvernement pour la création d'un comité central de pomologie, avee jardin d'expériences, appelé, comme il le disait lui-même, à rendre un service réel aux amateurs, en formant une collection type, étudiée sévèrement, eu égard à la elassification des fruits et à leur synonymie; et en réunissant, sous la direction d'une association spéciale, tous les arbres de semis provenant des pépinières Van Mons et Bivort, ainsi que les autres bons fruits cultivés soit en Belgique, soit à l'étranger, dont il pourrait être utile, après examen, de propager la eulture. C'est ce qui donna naissance à la Société Van Mons et aux Anrules de pomologic publiées par le comité central dont feu notre ami Royer était le président.

Ce fut encore sur ses instances que se formèrent les écoles d'horticulture dont il présidait annuellement les examens; et ce fut à son initiative et à sa volonté inébranlable lorsqu'il entrevoyait le bien, que nous devons l'existence de notre belle et utile institution et la direction si intelligente qui lui fut donnée dès le principe. Chacun de vous, messieurs, se rappellera encore cette première réunion qui eut lieu à Malines, le $\mathbf{3}$ mai 1859 et dans laquelle feu notre eher président a fait preuve de eet esprit de conciliation qui le distinguait, lorsqu'il proclamait en principe que la fédération projetée ne devait pas être une absorption de l'activité individuelle de chacune des Sociètés fédérées, mais que toutes devaient conserver leur attonomie et n'apporter au fonds commun que les améliorations constatées par chacune d'elles.

Enfin son activité ne connut jamais de bornes, et successivement pressident de la section agricole de Namur et vice-président de la Société agricole et forestière de la province de ce nom, puis président fondateur de la Société royale d'horticulture de la même province et président du premier congrès de pomologie, qu'il sut si parfaitement bien organiser en Belgique, il ne cessa de faire preuve d'une aptitude el d'une ardeur extraordinaires pour le travail, témoin les Annales de pomologie, les plublications de la Société van Mons et tant d'autres écrits disséminés dans diverses publications.

Messieurs, ce ne fut pas seulement au service de l'agrieulture et de l'horticulture qu'il sut meltre sa remarquable intelligence et son indomptable activité. Celles-ci se sont étendues à d'autres industries plus im- 
portantes encore, parmi lesquelles nous citerons surtout l'établissement de Floreffe dont il était l'âme et le président, ainsi que les verreries d'Herbatte qui se trouvent actuellement sous la puissante association des Verreries Namuroises.

Partout on fit appel à son patriotisme et à ses connaissances variées, et la chambre de commerce de Namur et le conseil provincial le comptaient parmi leurs membres les plus distingués. Feu notre Roi vénéré, qui savait si bien apprécier les hommes, a voulu récompenser les services rendus par notre laborieux président, en le nommant chevalier de son ordre.

Comme vous le voyez, Messieurs, Auguste Royer appartient à cette phalange d'lommes de progrès qui aidèrent si puissamment au développement de grandes entreprises; - d'une intelligence d'élite, aucun sujet ne lui était entièrement étranger; agriculture et horticulture, administration et finances, sciences et industries, - tout lui était familier, et sa persérérante activité sut triompher partout et toujours des obstacles inhérents à toute nouvelle entreprise.

Messieurs, avant de terminer, permettez-noi de déposer ici un regret tout personnel, c'est celui de n'avoir pu me joindre à la famille de notre cher défunt, et à ses nombreux amis, pour lui payer mon tribut d'hommages et déposer sur sa tombe, au nom de cette Fédération qu'il a créée, l'expression de nos bien vifs et bien sincères regrets. Malheureusement l'exposition universelle de Paris y a mis obstacle, mais j'ai été heureux d'apprendre par les journaux belges, qu'un de nos honorables collègues, notre ami Kegeljan, avait bien voulu se charger de cette douloureuse, mission. - Qu'il veuille en agréer ici notre sincère reconnaissance, car nous partageons tous les sentiments que, dans cette triste circonstance, il a si bien et si éloquemment exprimés.

Après un tel hommage rendu à la mémoire de notre cher président j'aurais pu me dispenser de prendre la parole, ou au moins j'aurais pu me borner à rendre hommage à ses connaissances aussi variées que profondes et à vous signaler ainsi l'étendue de la perte que nous venons de faire; mais il.m'eût été bien difficile, en occupant ce fauteuil qu'il honora pendant plusieurs années, de ne point me rappeler l'ami que nous avons perdu; il m'eût été bien difficile, Messieurs, d'étouffer les douloureuses impressions que j'éprouve en ce moment - car je sens qu'en ces cruelles épreuves de la séparation qui ne se multiplient que trop souvent à mon âge, je sens qu'il y a au fond du cœur une sensation bienfaisante, un sentiment de suave tristesse, je dirais même une profonde consolation à parler de ceux que nous avons aimés, à nous entretenir de leurs mérites, et à nous fortifier nous-mêmes par le souvenir du bien qu'ils on t fait et de la fermeté avec laquelle ils ont traversé les épreuves de la vie dont nous supportons le poids après eux.

Que ne devrais-je donc pas ajouter encore, s'il m'était permis de vous 
entretenir de tant d'affections qui viennent hélas! d'être brisées ? - La vie de famille fut tout pour lui, elle seule fit tout son bonheur et elle le soutint bicn souvent pendant ses longues années de souffrances où chaque jour il sentait le progrès du mal qui le conduisait au tombeau. Enfin Dieu le rappela à lui - et il rendit le dernier soupir au milieu des consolations de la religion et des soins affectueux et dévoués de sa courageuse famille. Il a succombé, Messieurs, à une maladie dont la marche lente et douloureuse a déjoué tous les efforts de l'art et toutes les tentatives de dévouement.

a Vous tous, qui avez su apprécier tout ce qu'il y avait de bonté et de générosité dans son cœur; - de loyauté et de noblesse dans son caractère, - vous qui connaissez tout ce qu'Auguste Royer a fait pour l'horticulture et l'activité sans borne qu'il n'a cessé de déployer pendant sa longue carrière - vos sentiments sont les miens, les miens sont les vôtres, et j'ose me dire votre organe en consignant dans nos annales, au nom de la Fédération et de toutes les Sociètés d'horticulture fédérées, avec l'cxpression du sentiment d'affectueuse estime que nous étions heureux de lui porter, celle de nos bons, vifs et bien sincères regrets.

a Inspirons-nous de son exemple - exemple fécond d'une vie consacrée tout entière aux intérêts de son pays - et que son souvenir souvenir impérissable d'un ami affectueux et dévoué - souvenir de l'homme de bien, du bon ciloyen, du chrétien résigné à la volonté de Dieu - ne s'efface jamaîs de nos cœurs et lui assure, parmi nous, le culte toujours dù à ceux qui, comme lui, ont parcouru une aussi' belle carrière. $p$

L'assemblée, après avoir applaudi cet éloquent panégyrique, a décidé qu'une souscription publique serait ouverte pour frapper une médaille à l'effigie d'Auguste Royer. Un de nos meilleurs graveurs, M. Léopold Wiener, est chargé de l'exécution de ce travail.

Royer laisse à une famille nombreuse, dont il était le chef vénéré, un nom entouré de l'estime générale : il laisse à son pays les résultats d'une puissante activité consacrée tout entière au bien public.

Éd. Morren. 





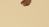




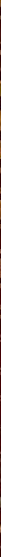

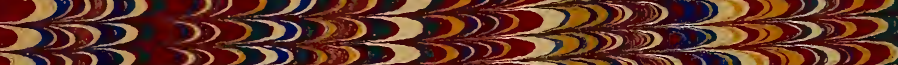

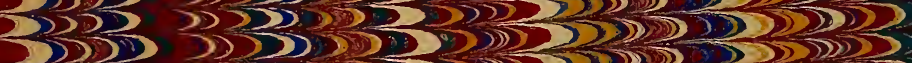

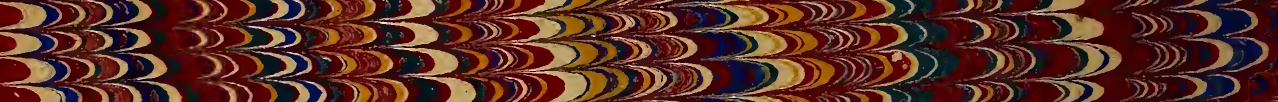

D) $=303252$

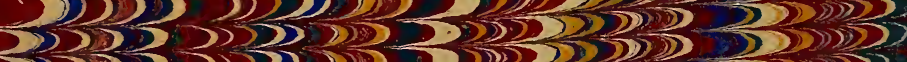
332532520 325353535

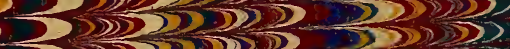
DiD 25 33 $250 \div 2$ $-2 D 25$ (D) 2252013 3523532

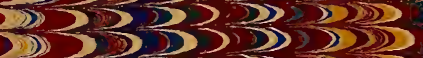
(1) 3233235

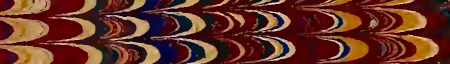
1202020 $320 \frac{2}{2} 2025$

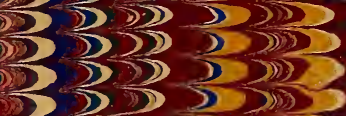

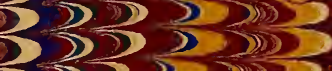
(ग) 250325 (3) 2202 3320 D) 25 352 232 325202020

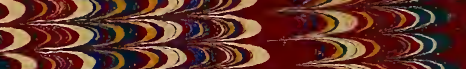
$35 D$ D.) 2

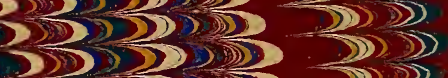
(2) $2 D$
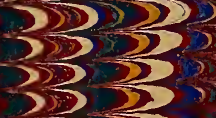
$\sum_{1} \sum_{2}^{2}$ $3 \sum_{3} 32302$ 2525393 32532502 23.2 3

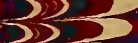

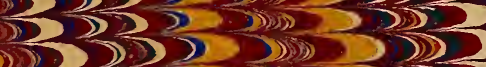
1) 333532 232353253 (i) 35352535

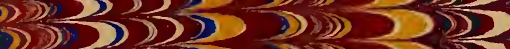

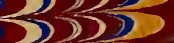
()) $)>3,20$

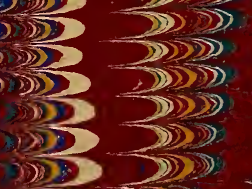
$\sum_{2} \sum_{\infty} \sum_{\infty}$ $\sum_{\infty} \sum_{20}^{\infty} \sum_{2}$

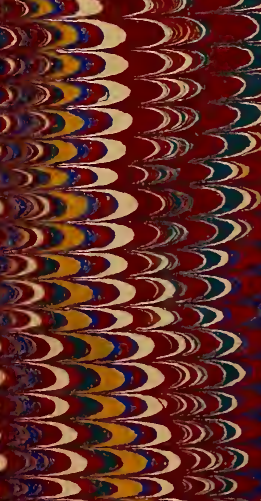
2325 DDD 3253

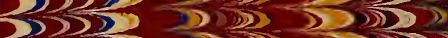
32202 3) 320152

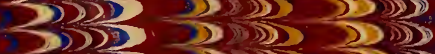

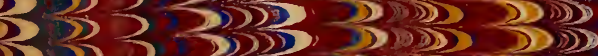
3) $>15305$ Ds (D) $D(2, D)(2,5)$

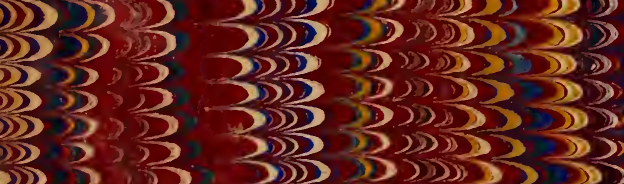

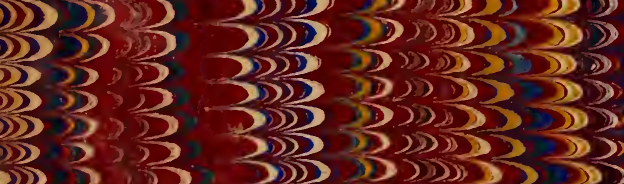

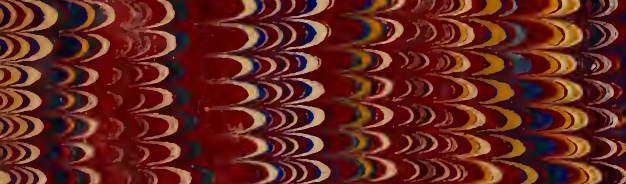

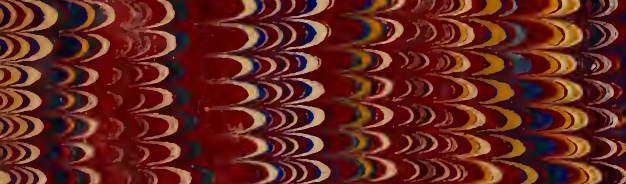

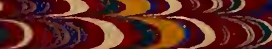
$3,230,3$ $3 x \geqslant 3$ 35323 32013 32325

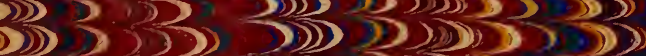
D)

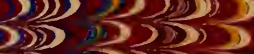

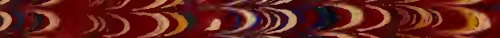
D $\gg 2 D$ (D) 3,035 5) 3 23 393535353 ग) (3) D D ग 3 3) $>3$ D) $>D S D$ (D) $>D D$

D) $D 2$ (2) 250

2520

$3>202$

3.20

325 3200 25

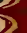

223 (D) 3 35 3

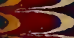
$3>25$ (4) (D) D D

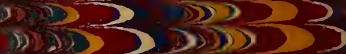
23 5

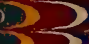
. D) D) D $D$

3 5) 5 in 5 15305 5 D) 23 (D) $)$ (3) (2) 12 (5) 32 5) 2 ()) D) 3 D) (3) D) $>3$ 


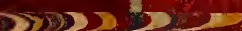

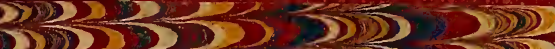

32525325 532523

(2) 123

323232

(ii) 3232

532523

321020

35,2532

520202

32502320

- S STD2 250

SDDSS $>2$

23,322

3,1025

353025 302 3) 350320 (ग) DD

(D) DDD

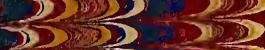
32525 () 2520 (3) DIDS SDI DSND SD 351000 $2 \div 3,203$ $\frac{3}{3}(35) 202023$ $5(20103$

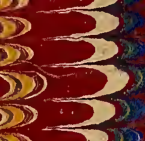

D $350203 \times 2$ $2 \ldots 2132$

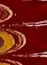

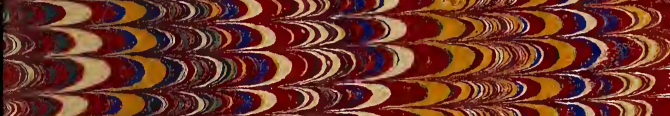

$3.25) 2325$

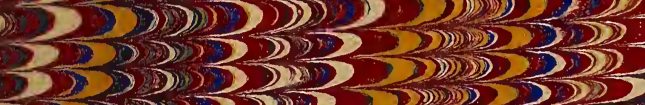

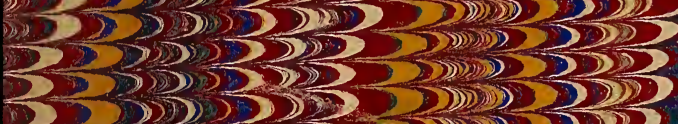
D) 252512002 3252537202

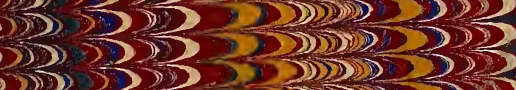

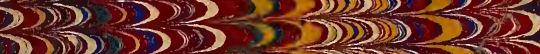
(D)D $D D(20) D D D(39)$ 320 350 $3>3$

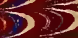
( SDDDDD 505

$3(2,2) D D D 25$

D) 325203 $(20) D D 25$ (5)

\section{(1)}

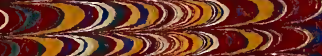

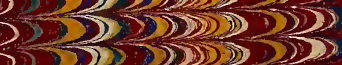

32015001050

(5) 2

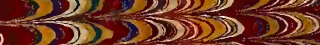

D) 22

D)

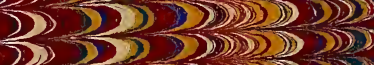

D) 25 (D) $D S>2$ 3) 12 D) $) 2$ $5 D 203$ 35202

$2 D 205$

(D)D $D$

2) 1202

23025

\section{$\infty 20$}

$22(20) 212$

\section{3) $)$}

3 y y $>$ r.

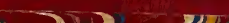

-

2

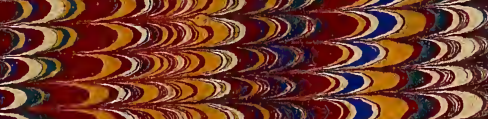

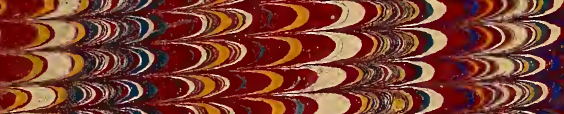

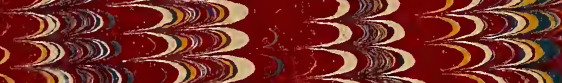
32025252 3503525252 ? $\gg 2$

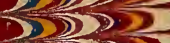

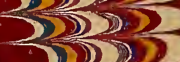
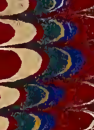

2525

(5) $\gg 2$ 38 ? 1) $) 12$

\section{.}

.

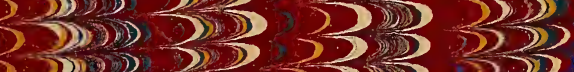

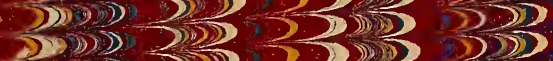
22050250253

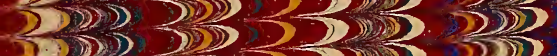
2013

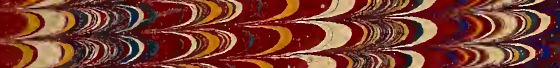
5) 35

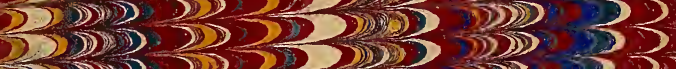

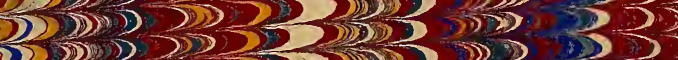

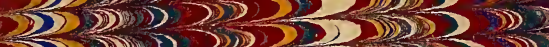

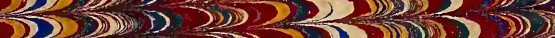

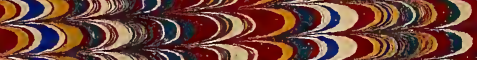
$\sum_{i \rightarrow \infty} \sum_{3}$ (ग) $>252535$

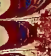




\section{LIBRARY OF CONGRESS}

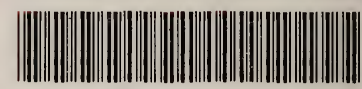

000276244 I8 